\title{
The first stars: a classification of CEMP-no stars
}

\author{
André Maeder and Georges Meynet
}

\author{
Geneva Observatory, Geneva University, 1290 Sauverny, Switzerland \\ e-mail: [andre.maeder;georges.meynet]@unige.ch
}

Received 1 April 2015 / Accepted 13 May 2015

\section{ABSTRACT}

\begin{abstract}
Aims. We propose and apply a new classification for the CEMP-no stars, which are carbon-enhanced metal-poor stars with no overabundance of s-elements and with $[\mathrm{Fe} / \mathrm{H}]$ generally $\leq-2.5$.

Methods. This classification is based on the changes in abundances for the elements and isotopes involved in the $\mathrm{CNO}, \mathrm{Ne}-\mathrm{Na}$, and $\mathrm{Mg}-\mathrm{Al}$ nuclear cycles. These abundances change very much owing to successive back and forth mixing motions between the He- and H-burning regions in massive stars (the "source stars" responsible for the chemical enrichment of the CEMP-no stars).

Results. The wide variety of the ratios $[\mathrm{C} / \mathrm{Fe}],{ }^{12} \mathrm{C} /{ }^{13} \mathrm{C},[\mathrm{N} / \mathrm{Fe}],[\mathrm{O} / \mathrm{Fe}],[\mathrm{Na} / \mathrm{Fe}],[\mathrm{Mg} / \mathrm{Fe}],[\mathrm{Al} / \mathrm{Fe}],[\mathrm{Sr} / \mathrm{Fe}]$, and $[\mathrm{Ba} / \mathrm{Fe}]$, which are the main characteristics making the CEMP-no and low-s stars so peculiar, is described well in terms of the proposed nucleosynthetic classification. We note that the $[(\mathrm{C}+\mathrm{N}+\mathrm{O}) / \mathrm{Fe}]$ ratios significantly increase for lower values of $[\mathrm{Fe} / \mathrm{H}]$.

Conclusions. The classification of CEMP-no stars and the behavior of $[(\mathrm{C}+\mathrm{N}+\mathrm{O}) / \mathrm{Fe}]$ support the presence, in the first stellar generations of the Galaxy, of fast-rotating massive stars experiencing strong mixing and mass loss (spinstars). This result has an impact on the early chemical and spectral evolution of the Galaxy.
\end{abstract}

Key words. stars: abundances - stars: Population III - stars: massive - stars: low-mass - stars: evolution - Galaxy: evolution

\section{Introduction}

Carbon-enhanced metal-poor (CEMP) stars are old low-mass stars with very low contents in iron and in other $\alpha$-elements (with atomic mass number $A>24$ ) that also show impressively large excesses of carbon, as well as generally of nitrogen and oxygen. This class of very interesting objects that belong to the first stellar generations in the Universe was initially recognized by Beers et al. (1992). Several subclasses of CEMP stars were distinguished and received an appropriate nomenclature in a review of the very metal-poor stars in the Galaxy (Beers \& Christlieb 2005). There are the so-called CEMP-s, CEMP-r/s, CEMP-r, and CEMP-no stars based on the contents in s- (particularly $\mathrm{Ba}$ ) and r-elements (particularly $\mathrm{Eu}$ ), or on the relative absence of these elements in the case of CEMP-no stars. For recent catalogs, the reader may refer to Masseron et al. (2010), Allen et al. (2012), Norris et al. (2013), and Hansen et al. (2015).

The CEMP-no stars dominate in the range of the lowest $[\mathrm{Fe} / \mathrm{H}]$ ratios, below $[\mathrm{Fe} / \mathrm{H}] \approx-3.0$ (Norris et al. 2013). Some stars with low s content are sometimes called "low-s stars" and may be related to the subclass of CEMP-no stars. Since several CEMP-no stars are MS or subgiant stars, their very peculiar CNO contents are likely not due to self-enrichment, but may result from the yields of previous objects called the "source stars", which are supposed to be among the first stars in the Universe.

What kinds of objects were these first stars? To get an answer, we have to do careful detective work to understand the origin of these very peculiar chemical abundances of CEMP-no stars. This is a fascinating topic that is closely related to nuclear astrophysics. Many models have been proposed to try to explain the chemical properties of CEMP-no stars, such as the discussions by Nomoto et al. (2013). In a recent work (Maeder et al. 2015, Paper I), we provide tests showing that the CNO abundances of CEMP-no stars result from the products of He-burning
( $\mathrm{C}$ and $\mathrm{O}$ ) that have gone through partial mixing and $\mathrm{CNO}$ processing in the H-burning shell, before being ejected to enrich the local interstellar medium. This is supported by the study of the ${ }^{12} \mathrm{C} /{ }^{13} \mathrm{C}$ ratios, of the $[\mathrm{C} / \mathrm{N}]$ and $[\mathrm{O} / \mathrm{N}]$ ratios. In CEMP-no stars, the elements involved in the $\mathrm{CNO}$ cycle cover a completely different range of variations than the $\alpha$-elements (with an atomic mass number higher than 24). In addition, the heavy elements $\mathrm{Na}, \mathrm{Mg}$, and $\mathrm{Al}$ participating in the $\mathrm{Ne}-\mathrm{Na}$ and $\mathrm{Mg}-\mathrm{Al}$ cycles behave like the CNO elements and not like the $\alpha$-elements, which is quite consistent with the operation of $\mathrm{H}$-burning in massive stars.

Our purpose here is to examine some further consequences of the general model of fast-rotating massive stars (spinstars) with mixing and mass loss (Meynet et al. 2006; Maeder \& Meynet 2012), which we applied to CEMP-no stars in Paper I. This model accounts for the wide variety of the typical overabundances of $\mathrm{C}, \mathrm{N}, \mathrm{O}, \mathrm{Na}, \mathrm{Mg}, \mathrm{Al}$, and of s-elements in some cases, as well as for some specific relations between these elements (cf. Paper I). Section 2 proposes detailed classification criteria. In Sect. 3, we apply this classification to the whole sample of known CEMP-no stars. Section 4 gives the conclusions.

\section{Criteria for the classification of CEMP-no stars}

The classification is based on the nuclear processes that have occurred in the source stars and on the possible release by mass loss of the mixture produced at various successive steps of mild mixing. If the $\mathrm{C}$ and $\mathrm{O}$ elements resulting from He-core burning enter by mild mixing into the $\mathrm{H}$-burning shell, the nuclear reactions there build some new elements, such as ${ }^{13} \mathrm{C}$ and ${ }^{14} \mathrm{~N}$. Now, if these elements are again mildly mixed into the He-burning zone, ${ }^{13} \mathrm{C}$ and ${ }^{14} \mathrm{~N}$ will be destroyed and some other new elements may be created by $\alpha$ captures, such as ${ }^{20,22} \mathrm{Ne}$ and ${ }^{24,25,26} \mathrm{Mg}$, together with s-elements of the first peak, such as Sr and Y, and 
Table 1. Attempt at a classification of the CEMP-no stars based on the effects of successive mixing phases between the H- and He-burning zones and then mass loss from the source stars.

\begin{tabular}{|c|c|c|c|c|c|c|c|c|c|c|}
\hline Physics & Class & {$[\mathrm{Ba} / \mathrm{Fe}]$} & {$[\mathrm{Sr} / \mathrm{Fe}]$} & {$[\mathrm{Na} / \mathrm{Fe}]$} & {$[\mathrm{Mg} / \mathrm{Fe}]$} & {$[\mathrm{Al} / \mathrm{Fe}]$} & ${ }^{12} \mathrm{C} /{ }^{13} \mathrm{C}$ & {$[\mathrm{C} / \mathrm{N}]$} & {$[\mathrm{O} / \mathrm{N}]$} & {$\left[\frac{\mathrm{C}+\mathrm{N}+\mathrm{O}}{\mathrm{Fe}}\right]$} \\
\hline & \multicolumn{10}{|c|}{ EMP stars with none or very few heavy elements } \\
\hline $\begin{array}{l}\text { Initial abund. } \\
\text { Prod. of H-burn. }\end{array}$ & $\begin{array}{c}\mathbf{0} \\
\mathbf{0 +}\end{array}$ & \multicolumn{9}{|c|}{$\begin{array}{l}\text { Very low abundances of all heavy elements } \\
\text { He-, light }{ }^{13} \mathrm{C}-\text {, and }{ }^{14} \mathrm{~N} \text {-enrichments possible if metallicity } Z>10^{-9}\end{array}$} \\
\hline \multicolumn{11}{|c|}{ CEMP stars with products of He-burning, without $H$ (objects like WC and WO stars) ${ }^{a}$} \\
\hline He-bur & 1 & low & low & low & low & low & $\infty$ & $\infty$ & $\infty$ & very high \\
\hline He-burn.(WO s & $1+$ & \multicolumn{9}{|c|}{ Same, with less ${ }^{12} \mathrm{C}$, more ${ }^{16} \mathrm{O},{ }^{20,22} \mathrm{Ne}$ present, maybe some ${ }^{24} \mathrm{Mg}$} \\
\hline & \multicolumn{10}{|c|}{ CEMP-no stars with no s-elements and generally no $M g$ and $A l^{b}$} \\
\hline $\begin{array}{l}\text { Mild 2nd H-burn. } \\
\text { Strong 2nd H-burn. }\end{array}$ & $\begin{array}{c}2 \\
2+\end{array}$ & $\begin{array}{c}\sim 0 \\
\text { low }\end{array}$ & $\begin{array}{c}\sim 0 \\
\text { low }\end{array}$ & $\begin{array}{l}\text { low } \\
\text { low }\end{array}$ & $\begin{array}{l}\text { low } \\
\text { low }\end{array}$ & $\begin{array}{l}\sim 0 \\
\text { low }\end{array}$ & $\begin{array}{l}\text { low-mid } \\
\text { low }\end{array}$ & $\begin{array}{l}\geq 0 \\
\leq 0.2\end{array}$ & $\begin{array}{l}\geq 0 \\
\leq 0.3\end{array}$ & $\begin{array}{l}\text { low-mid } \\
\text { low-mid }\end{array}$ \\
\hline & \multicolumn{10}{|c|}{ CEMP stars with s-elements possible, $(C E M P-n o, l o w-s, C E M P-s)^{c}$} \\
\hline $\begin{array}{l}\text { Mild 2ndHe-burn. } \\
\text { Strong 2ndHe-burn. } \\
\text { More n-emission }\end{array}$ & $\begin{array}{c}3 \\
3+ \\
3++ \\
\end{array}$ & $\begin{array}{l}\text { low-mid } \\
\text { mid } \\
\text { high }\end{array}$ & $\begin{array}{l}\text { low-mid } \\
\text { high } \\
\text { high }\end{array}$ & $\begin{array}{l}\text { low } \\
\text { any } \\
\text { any }\end{array}$ & $\begin{array}{l}\text { low-mid } \\
\text { mid-high } \\
\text { high }\end{array}$ & $\begin{array}{l}\text { low } \\
\text { any } \\
\text { any }\end{array}$ & $\begin{array}{l}\text { high } \\
\text { high } \\
\text { high }\end{array}$ & $\begin{array}{c}\text { mid-high } \\
>0.3 \\
>0.3\end{array}$ & $\begin{array}{l}\text { mid-high } \\
\quad>0.4 \\
>0.4\end{array}$ & $\begin{array}{l}\text { mid-high } \\
\text { mid-high } \\
\text { high }\end{array}$ \\
\hline \multicolumn{11}{|c|}{ CEMP stars with s-elements possible, $(C E M P-n o, \text { low-s, CEMP-s })^{d}$} \\
\hline $\begin{array}{l}\text { H-burn. of Class } 3 \\
\text { H-burn. of Class } 3+ \\
\text { H-burn. of Class } 3++\end{array}$ & $\begin{array}{c}4 \\
4+ \\
4++ \\
\end{array}$ & $\begin{array}{l}\text { mid } \\
\text { mid } \\
\text { high }\end{array}$ & $\begin{array}{l}\text { mid } \\
\text { high } \\
\text { high }\end{array}$ & $\begin{array}{l}\text { high } \\
\text { high } \\
\text { high }\end{array}$ & $\begin{array}{l}\text { mid } \\
\text { mid-high } \\
\text { high }\end{array}$ & $\begin{array}{l}\text { any } \\
\text { mid } \\
\text { high }\end{array}$ & $\begin{array}{l}\text { low-mid } \\
\text { low } \\
\text { low }\end{array}$ & $\begin{array}{l}\text { mid } \\
\leq 0.3 \\
\text { low }\end{array}$ & $\begin{array}{l}\text { mid } \\
\leq 0.4 \\
\text { low }\end{array}$ & $\begin{array}{l}\text { mid-high } \\
\text { mid-high } \\
\text { high }\end{array}$ \\
\hline
\end{tabular}

Notes. Enhancements in helium should also be present in CEMP stars. ${ }^{(a)}$ the existence of such stars is uncertain at extremely low $Z$, but products of 1 and $1+$ intervene in mixing. ${ }^{(b)} \mathrm{Ne}-\mathrm{Na}$ cycle may produce $\mathrm{Na}$ from $\mathrm{Ne}$, especially for $2+$, thus leading to Class $2+\mathrm{Na}$. Mg-Al cycle unlikely. ${ }^{(c)}$ If $\mathrm{Na}$ produced in stages 2, Stages $3 \mathrm{Na}, 3+\mathrm{Na}, 3++\mathrm{Na}$ may result. $\mathrm{Mg}$ present, but $\mathrm{Al}$ unlikely. ${ }^{(d)}$ Third passage in the $\mathrm{H}$-burning zone. $\mathrm{Na}$ always high. Burning intensity is increasing from 4 to $4++$. Further mild mixing also leads to $4++$, see text.

(depending on the conditions) even of the second peak, such as $\mathrm{Ba}$ and $\mathrm{La}$. These products may continue to undergo mixing and go back to the H-burning region, where ${ }^{13} \mathrm{C}$ and ${ }^{14} \mathrm{~N}$ are built again. Now the $\mathrm{Ne}-\mathrm{Na}$ and $\mathrm{Mg}-\mathrm{Al}$ cycles may operate, producing $\mathrm{Na}$ and $\mathrm{Al}$. At each step, mass ejection may occur, preserving the actual nucleosynthetic products.

In the sequence of successive back-and-forth motions between the He- and H-burning regions, some elements are more or less destroyed, while others are synthesized. This creates the wide variety of the observed ratios, such as ${ }^{12} \mathrm{C} /{ }^{13} \mathrm{C},[\mathrm{C} / \mathrm{Fe}]$, [N/Fe], [O/Fe], [Na/Fe], [Mg/Fe], [Al/Fe], [Sr/Fe], and [Ba/Fe]. These successive stages of mixing have chemical abundances different from the case of full or convective mixing, which would lead to the equilibrium abundance ratios that essentially correspond to the highest temperature in the convective region. The processes of mild mixing produce large differences between the abundances of the mentioned elements and of the $\alpha$-elements with $A>24$. Thus, depending on the history of mass loss, very different chemical yields may be produced. In the line of the results of Paper I, we establish here the classification criteria and apply them to individual stellar observations.

Table 1 shows the proposed classification criteria for CEMPno stars. The first column mentions the main physical processes involved, the second one assigns a class or a step in the backand-forth motions between the He- and H-burning regions, and the next columns indicate the possible ranges for the significant abundances ratios defining the considered classes. We note that, on one hand, more subclasses might be needed to fully represent the complex effects of mixing and mass loss, and, on the other, some proposed classes might not contain some real objects. In any case, the comparison of the predicted classes of chemical compositions with the observations will show us whether the proposed scheme is valid.

\subsection{Classes 0 and 1}

The first kind of possible composition is the initial one, from which the source stars were formed. If these really belonged to the very first stellar generation, their composition should be the cosmological one of pure hydrogen and helium with tiny amounts of light elements, as for lithium with $A(\mathrm{Li})=$ $\log n(\mathrm{Li}) / n(\mathrm{H})+12=2.72$ (Cyburt et al. 2008). These would be extremely metal-poor stars (EMP). In fact, supernovae from massive stars may explode already after a few million years, so that the content of the first stellar generations in heavy elements may be slightly different from zero. This initial composition would correspond to Class or Step " 0 ". Class " $0+$ " is associated to CNO-burning of a medium with the above initial composition. For CNO reactions to be effective, the initial metallicity $Z$ should be above $\sim 10^{-9}$. The $0+$ class should be enriched in helium with respect to Class 0 and should exhibit low ${ }^{12} \mathrm{C} /{ }^{13} \mathrm{C}$ and $[\mathrm{C} / \mathrm{N}]$ ratios, if observable.

Another possible class is that of stars with none or very few Fe and $\alpha$-elements (with atomic mass number $A>24$ ) that would exhibit the pure products of partial He-burning, i.e. a mixture of $\mathrm{He}, \mathrm{C}$, and $\mathrm{O}$, like what the WC stars show. These stars would contain no hydrogen. We call it composition Class " 1 ". We do not know whether the extremely low-metallicity populations contain such stars. The extreme case we know at present is that of I Zw 18 with a metallicity $Z \approx 3 \times 10^{-4}$ (Lebouteiller et al. 2013), where numerous Wolf-Rayet stars seem to be present (Crowther 2010). Formally, we could also consider a Class " $1+$ ", where the products of He-burning correspond to an advanced stage of He-burning. It would be characterized by a low-tomoderate amount of $\mathrm{C}$, with a large content of $\mathrm{O}$, together with some ${ }^{20} \mathrm{Ne}$ (and maybe tiny amounts of ${ }^{24} \mathrm{Mg}$ ) produced by $(\alpha, \gamma)$ captures on ${ }^{16} \mathrm{O}$ and a very small amount of ${ }^{22} \mathrm{Ne}$ resulting 


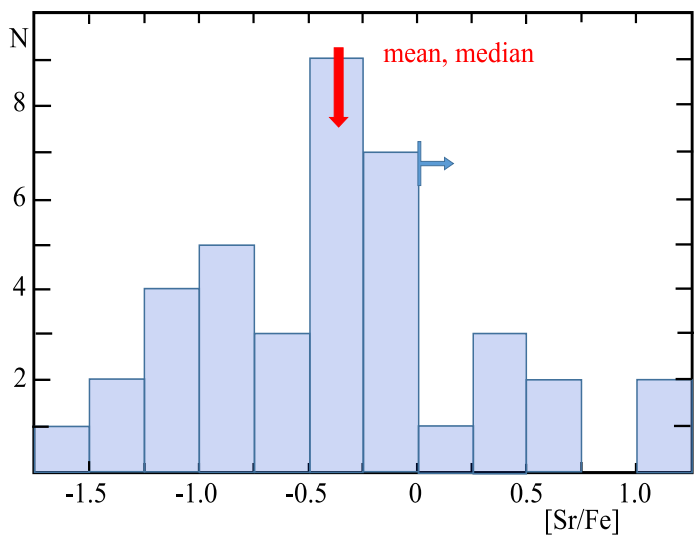

Fig. 1. Distribution of the ratio [ $\mathrm{Sr} / \mathrm{Fe}]$ for the CEMP-no stars in Table 2. The positions of the mean and median values are indicated. The small vertical bar with an arrow on the right indicates above which value we consider that there are significant excesses.

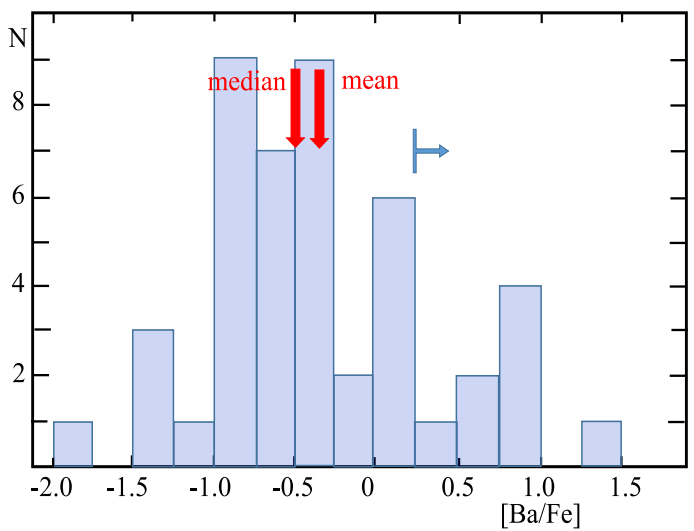

Fig. 2. Distribution of the ratio $[\mathrm{Ba} / \mathrm{Fe}]$ for the CEMP-no stars in Table 2. Same remarks as for Fig. 1.

from the N-destruction. This sort of object would be like the rare WO stars, which are often observed in relatively low $Z$ regions.

\subsection{Classes 2}

These classes are formed by source stars in which products of He-burning (mainly $\mathrm{C}$ and $\mathrm{O}$ ) have been partially mixed and processed by the $\mathrm{CNO}$ cycles in the H-burning shell, creating significant amounts of ${ }^{14} \mathrm{~N}$. The $\mathrm{CNO}$ elements transported by mixing to the surface of the source stars may be lost by stellar winds further, for example in the red supergiant stage as shown by Meynet et al. (2006) or by instabilities just before the supernova explosion (Moriya \& Langer 2015). Some CEMP-no stars are likely formed from a medium significantly enriched in such ways. This corresponds to Classes 2 and $2+$, the first one having very mild H-burning, while the second shows more intense $\mathrm{H}$-burning. As indicated in Table 1, such objects are expected to have no or very small amounts of s-elements like $\mathrm{Sr}$ or Ba. The elements $\mathrm{Na}$ and $\mathrm{Al}$ produced by the $\mathrm{Ne}-\mathrm{Na}$ and $\mathrm{Mg}-\mathrm{Al}$ cycles (Maeder 2009) should also be generally very low or absent. In Class $2,[\mathrm{C} / \mathrm{N}]$ and $[\mathrm{O} / \mathrm{N}]$ still have values above some level, say about 0 , as a result of mild $\mathrm{CNO}$ processing. At the same time, the ratio ${ }^{12} \mathrm{C} /{ }^{13} \mathrm{C}$ is low to medium, say $\leq 10$ (see, for example, Fig. 1 in Paper I). Strong CNO processing (Class 2+) produces very low ${ }^{12} \mathrm{C} /{ }^{13} \mathrm{C}$ ratios, as well as $[\mathrm{C} / \mathrm{N}]$ and $[\mathrm{O} / \mathrm{N}]$ ratios below the means, which are 0.20 and 0.27 , respectively.

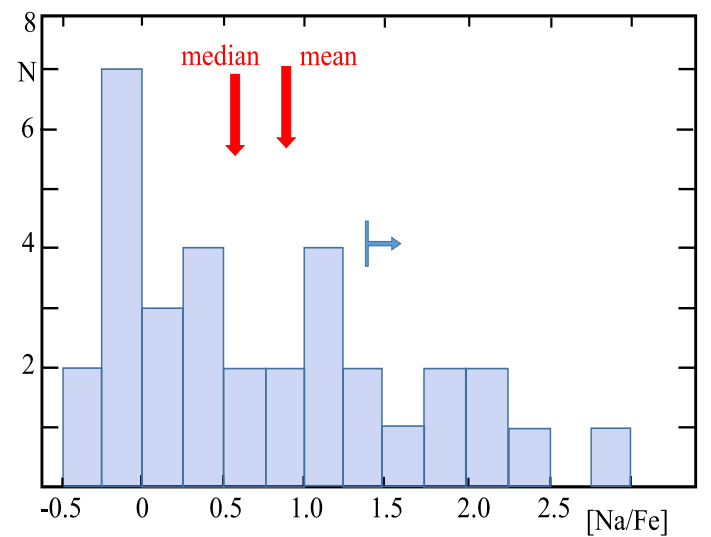

Fig. 3. Distribution of the ratio $[\mathrm{Na} / \mathrm{Fe}]$ for the CEMP-no stars in Table 2. Same remarks as for Fig. 1.

If ${ }^{20} \mathrm{Ne}$ has been produced by core He-burning in sufficiently hot objects, mixing may bring some amount of it into the $\mathrm{H}$-burning region, allowing the $\mathrm{Ne}-\mathrm{Na}$ cycle to operate and thus to produce some ${ }^{23} \mathrm{Na}$. This situation is more likely in the case of strong nuclear processing, this would lead to a Class $2+\mathrm{Na}$. The creation of some ${ }^{24} \mathrm{Mg}$, if any at all, is very low in the Heburning phase, thus strong signatures of the subsequent $\mathrm{Mg}-\mathrm{Al}$ cycles are generally not expected in Classes 2 and 2+. However, some limited operations of the $\mathrm{Ne}-\mathrm{Na}$ and $\mathrm{Mg}-\mathrm{Al}$ cycles may contribute to the scatter of the values as illustrated by the Figs. 3 to 5. Finally, we note that the $[(\mathrm{C}+\mathrm{N}+\mathrm{O}) / \mathrm{Fe}]$ ratios should lie on the low-to-medium side of the distribution illustrated in Fig. 7, i.e., smaller than about 2.5.

\subsection{Classes 3}

The products resulting from Classes 2 and $2+$ may again be mixed into the He-burning core (or in the He-burning shell in cases of advanced stages). This leads to the synthesis of new elements and makes Classes 3, 3+, and 3++. Successive $\alpha$-captures by ${ }^{14} \mathrm{~N}$ produce ${ }^{22} \mathrm{Ne}$. Further reactions may also occur: ${ }^{22} \mathrm{Ne}(\alpha$, $n)^{25} \mathrm{Mg}$, and ${ }^{22} \mathrm{Ne}(\alpha, \gamma)^{26} \mathrm{Mg}$. The free neutrons emitted by the first one may lead to producing some s-elements of the first peak, such as Sr and Y (Frischknecht et al. 2010). For that it is necessary for some heavy elements of the Fe peak to be present. This is the case, since all CEMP-no stars exhibit iron, except perhaps SMSS 0313-6708, where only an upper limit is given by Keller et al. (2014). In case of intense neutron emission or when the number of free neutrons per seed is high, some s-elements of the second peak may also be synthesized, for instance Ba and La. In any case, even if weak, the reactions may contribute to the scatter of the abundances (Figs. 1 and 2). Simultaneously, the successive $\alpha$ captures operate and lead to the beginning of the sequence of $\alpha$-elements: ${ }^{12} \mathrm{C} \rightarrow{ }^{16} \mathrm{O} \rightarrow{ }^{20} \mathrm{Ne}$.

The presence of nitrogen and especially of ${ }^{13} \mathrm{C}$ in all CEMPno stars implies that the mixing is always partial, otherwise in case of full mixing these elements would be destroyed. We define Class 3 (cf. Table 1) as the case of a mild amount of processing into the He-burning zone, creating more ${ }^{12} \mathrm{C},{ }^{16} \mathrm{O}$, and ${ }^{14} \mathrm{~N}$, leading to ${ }^{22} \mathrm{Ne}$ and few other $\alpha$-captures. Thus, objects in this class show none or few s-elements. The He-burning favors midto-high $[\mathrm{C} / \mathrm{N}],[\mathrm{O} / \mathrm{N}]$ ratios and a relatively high $(>10){ }^{12} \mathrm{C} /{ }^{13} \mathrm{C}$ ratio (within the range of CEMP-no stars). At the same time, the $[(\mathrm{C}+\mathrm{N}+\mathrm{O}) / \mathrm{Fe}]$ ratios are on the average slightly higher than in Class 2, mostly between 2 and 3 . 
Table 2. Abundance data for the sample stars considered in the attempt to assign a class.

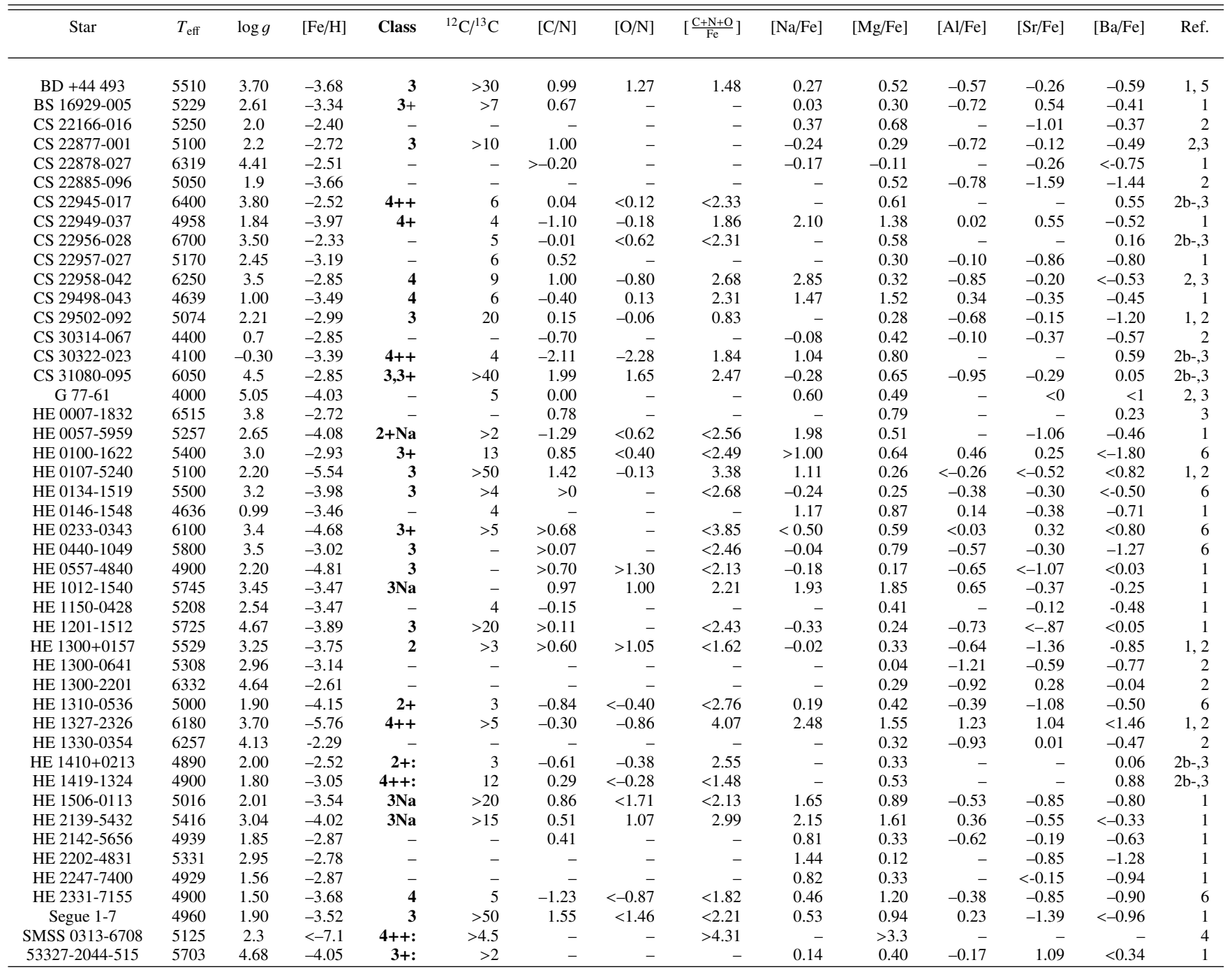

References. Norris et al. (2013); 2) Allen et al. (2012); 3) Masseron et al. (2010); 4) Keller et al. (2014) give values of [Li/H], [C/H], [Mg/H], and $[\mathrm{Ca} / \mathrm{H}]$ and upper limits in the case of other elements for this star; 5) Ito et al. (2013) give a lower limit for ${ }^{13} \mathrm{C} /{ }^{12} \mathrm{C}$; 6) Hansen et al. (2015).

We define stars of Class $3+$ as those stars where the process of He-burning looks more advanced than in Case 3. The criteria are indicated in Table 1 . The reaction producing ${ }^{25} \mathrm{Mg}$ leads to some significant amount of s-elements of the first peak, typically strontium Sr. In this context, we recall that for some time, the occurrence of Sr in very low-metallicity stars was challenging theoretical understanding (Frebel et al. 2005). Figure 1 shows the present distribution of the ratios $[\mathrm{Sr} / \mathrm{Fe}]$ in the CEMPno stars. This distribution shows a rather centered peak, nevertheless with some asymmetry due to a long tail on the right. For the moment, we consider a star in Class $3+$ if its value $[\mathrm{Sr} / \mathrm{Fe}]$ is $\geq 0.0$. At the same time, the star should present some mediumto-high abundance of magnesium. In Class $3++$, the He-burning is even more advanced than above, and the emission of neutrons is enough to produce elements of the second s peak, typically barium $\mathrm{Ba}$. Figure 2 shows the distribution of the $[\mathrm{Ba} / \mathrm{Fe}]$ ratios for the stars of Table 2, which is rather similar to that of $[\mathrm{Sr} / \mathrm{Fe}]$. A value $[\mathrm{Ba} / \mathrm{Fe}] \geq \sim 0.25$ may correspond to Class $3++$.

If $\mathrm{Na}$ is present in the stages corresponding to Classes 2 (being produced by the $\mathrm{Ne}-\mathrm{Na}$ cycle operating from the $\mathrm{Ne}$ possibly synthesized in the core He-burning), it should then also be there in the corresponding classes $3 \mathrm{Na}, 3+\mathrm{Na}$, and $3++\mathrm{Na}$. As we have seen, some $\mathrm{Mg}$ is unavoidably present in Classes $3+$ and $3++$, as a result of $\alpha$ captures on ${ }^{22} \mathrm{Ne}$. However the occurrence of $\mathrm{Al}$ is unlikely since it is not created in the He-burning stage.

\subsection{Classes 4}

If the elements that have experienced the previous back and forth motions again enter the H-burning region, they may produce Classes 4, 4+, and 4++. These three classes result from further CNO-burning of the elements corresponding to Classes 3, $3+$, and $3++$, respectively (with or without $\mathrm{Na}$ ). In Class 4 there are only small amounts of s-elements, CNO-burning reduces the ${ }^{12} \mathrm{C} /{ }^{13} \mathrm{C},[\mathrm{C} / \mathrm{N}]$, and $[\mathrm{O} / \mathrm{N}]$ ratios, while the sum $[(\mathrm{C}+\mathrm{N}+\mathrm{O}) / \mathrm{Fe}]$ remains unchanged. All classes of Group 4 may have high $[\mathrm{Na} / \mathrm{Fe}]$ as a result of the $\mathrm{Ne}-\mathrm{Na}$ cycle operating on the Ne isotopes created in the previous He-burning stages. Figure 3 illustrates the distribution of the $[\mathrm{Na} / \mathrm{Fe}]$ ratios. This distribution is rather flat and broad, which may result from the wide range of conditions leading to the $\mathrm{Na}$ synthesis. The $\mathrm{Mg}$ content is not modified with respect to Class 3 . The $\mathrm{Mg}-\mathrm{Al}$ cycle may be active 


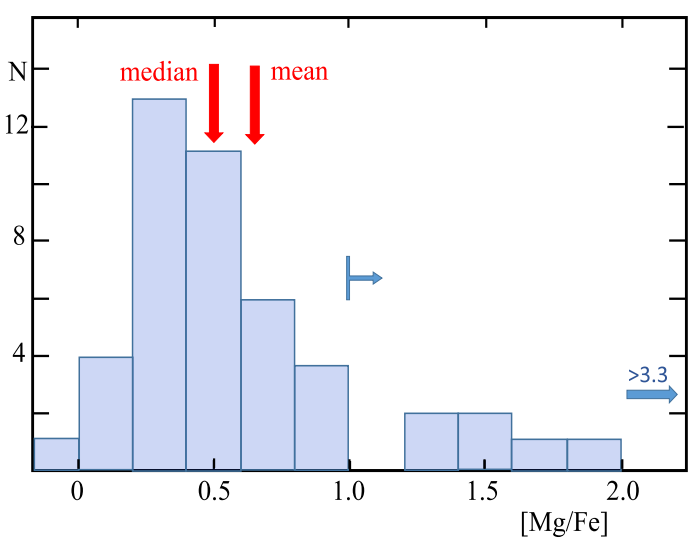

Fig. 4. Distribution of the ratio $[\mathrm{Mg} / \mathrm{Fe}]$ for the CEMP-no stars in Table 2. Same remarks as for Fig. 1. The blue arrow on the right indicates the star SMSS0313-6708 with a ratio $[\mathrm{Mg} / \mathrm{Fe}]>3.3$.

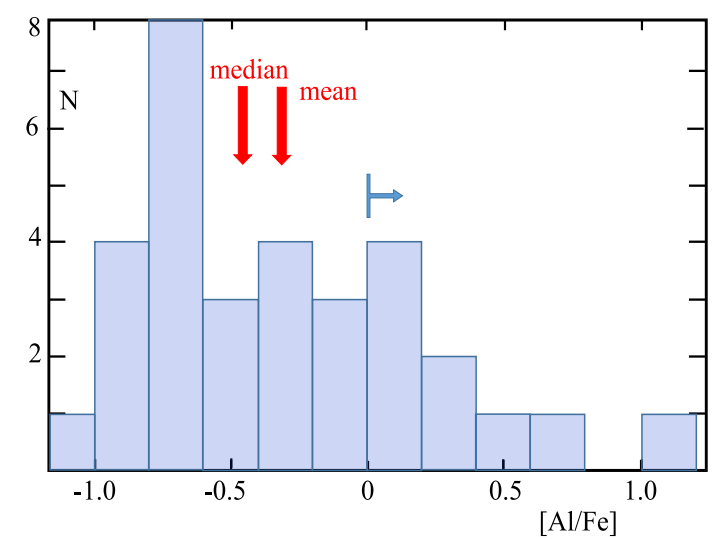

Fig. 5. Distribution of the ratio $[\mathrm{Al} / \mathrm{Fe}]$ for the CEMP-no stars in Table 2. Same remarks as for Fig. 1.

or not, so that any abundance of $\mathrm{Al}$ is possible. (The possible operation of the $\mathrm{Mg}-\mathrm{Al}$ cycle does not significantly modifies the Mg-content.)

Class 4+ results from further $\mathrm{H}$-burning of a medium of composition corresponding to Class $3+$. Thus, Sr shows significant excess, while this is not the case for $\mathrm{Ba}$. We have the usual effect of CNO-burning: low ${ }^{12} \mathrm{C} /{ }^{13} \mathrm{C},[\mathrm{C} / \mathrm{N}]$, and $[\mathrm{O} / \mathrm{N}]$ ratios. $\mathrm{Na}$ is high as before. We expect some moderate production of $\mathrm{Al}$ from the $\mathrm{Mg}-\mathrm{Al}$ cycle.

Class $4++$ presents both high abundances of $\mathrm{Sr}$ and $\mathrm{Ba}$, as a result of strong production of s-elements from intense $(\alpha, n)$ captures on ${ }^{22} \mathrm{Ne}$. In addition, this class shows the above -mentioned effects of CNO-burning, as well as of the full operation of the $\mathrm{Ne}-\mathrm{Na}$ and $\mathrm{Mg}-\mathrm{Al}$ cycles, therefore the $[\mathrm{Na} / \mathrm{Fe}],[\mathrm{Mg} / \mathrm{Fe}]$, and $[\mathrm{Al} / \mathrm{Fe}]$ ratios are all high. In this respect, we note that the distributions of the $[\mathrm{Mg} / \mathrm{Fe}]$ and $[\mathrm{Al} / \mathrm{Fe}]$ ratios are similar (Figs. 4 and 5), which is consistent with the fact that these elements have a mother-daughter relation. The distribution of the $[\mathrm{Al} / \mathrm{Fe}]$ ratios is different from that of $[\mathrm{Na} / \mathrm{Fe}]$, which might be because the $\mathrm{Al}$ synthesis results from a narrower range of conditions than for $\mathrm{Na}$, since the typical temperature of the $\mathrm{Mg}-\mathrm{Al}$ cycle is higher than for the $\mathrm{Ne}-\mathrm{Na}$ cycle. Moreover, the $\mathrm{Mg}-\mathrm{Al}$ cycle requires the previous synthesis of $\mathrm{Mg}$, which also demands higher temperatures than for the $\mathrm{Ne}$ synthesis, which is necessary for the operation of the $\mathrm{Ne}-\mathrm{Na}$ cycle.

If mixing between the $\mathrm{H}$ - and He-burning regions occurs much more, the composition of the mixtures will also tend toward that of Class $4++$ with the possibility of a variety of $[\mathrm{C} / \mathrm{N}]$,
$[\mathrm{O} / \mathrm{N}]$, and ${ }^{12} \mathrm{C} /{ }^{13} \mathrm{C}$ ratios. These ratios would be more like those of Class $3++$ if He-burning was the last nuclear-burning experienced, or more like Class $4++$ if the mixture has just gone through H-burning. This last possibility is much more likely, since to be observed, matter has to escape from the source star and thus has to originate in the outer layers. As a matter of fact, we have not identified any $3++$ objects, while several stars of Class $4++$ are present in the sample without any ambiguity.

The CEMP stars with s-elements are generally classified as a group different from the CEMP-no stars. A consequence of the scheme proposed here is that some CEMP-s stars may be genetically connected to the CEMP-no stars, being formed in a sequence of similar processes and from stars with high initial masses. Thus, further analysis of the samples of CEMP-s stars may be useful in future to extract the objects that in fact belong to the genetic group of the so-called CEMP-no stars, likely stemming from massive stars in view of the short timescales associated to objects with $[\mathrm{Fe} / \mathrm{H}]$ lower than -3.0 (Chiappini et al. 2008; Matteucci 2012).

\subsection{A word of caution and specific remarks}

The rich composition variety of CEMP-no stars results, as we are suggesting, from mild mixing between the $\mathrm{H}$ - and He-burning regions of massive stars with mass loss, which allow the new elements to escape into the ambient medium. The range of possible initial stellar masses is wide, say all masses above about $10 M_{\odot}$. Thus, the reduction of the many complex processes occurring in stars of different masses, rotation velocities, mass loss, and metallicities to a limited number of simple classes is a kind of wager. We examine whether such a complex reality encounters any difficulty when being forced into such a simple scheme. As a matter of fact, problems rarely occur. We would also like to point out that the values of the limits between the various classes may change a bit when larger samples of CEMP-no stars become available and when observations become more accurate. Although mainly qualitative at present, the classification is nevertheless very constraining, owing to the number of criteria to be satisfied for each class. In future, it may become more quantitative, if progress in stellar models and observations allows it.

There is a fundamental difference between elements like $\mathrm{C}$, $\mathrm{N}, \mathrm{O}, \mathrm{Ne}, \mathrm{Na}, \mathrm{Mg}$, and $\mathrm{Al}$ and the s-elements. The first ones in CEMP-no stars are primary elements, (i.e., made from the initial $\mathrm{H}$ and $\mathrm{He}$ ), while the second ones are secondary elements. To be created, the s-elements require seed elements of the $\mathrm{Fe}$ peak, by which free neutrons are captured. The behavior of these two kinds of elements is different in models of galactic chemical evolution.

Most interestingly, Hansen et al. (2015) have found a "floor" - i.e., a plateau independent of $[\mathrm{Fe} / \mathrm{H}]($ for $[\mathrm{Fe} / \mathrm{H}]<\sim-3.0)-$ in the absolute $\mathrm{Ba}$ abundances of CEMP stars $A(\mathrm{Ba})=\log \epsilon(\mathrm{Ba})+$ $12.0 \approx-2.0$, where $\epsilon$ indicates the abundances in number. This result implies that for the lower $[\mathrm{Fe} / \mathrm{H}]$ ratios, the observed values of $[\mathrm{Ba} / \mathrm{Fe}]$ become increasingly higher. Here, we only have CEMP-no stars, i.e., those with no overabundance of Ba according to the formal definition. Nevertheless, in the limited range of $[\mathrm{Ba} / \mathrm{Fe}]$ ratios concerned (normally $[\mathrm{Ba} / \mathrm{Fe}]<1$ ), the data of Table 2 support this result. There are eight stars that show an excess of s-elements with [Sr/Fe] $>0$ (Fig. 1): BS 16929-005, CS 22949-037, HE 0100-1622, HE 0233-0343, HE 1300-2201, HE 1327-2326, HE 1330-0354, and 53327-2044-515. They all have negative values of $[\mathrm{Ba} / \mathrm{Fe}]$, except the two largely most metaldeficient ones, HE 0233-0343 and HE 1327-2326, where the upper limits are highly positive. 
How do we interpret the Ba floor found by Hansen et al. (2015)? We suggest the following. At the lowest $[\mathrm{Fe} / \mathrm{H}]$ values, the number of seeds per free neutron becomes small enough that the neutron flux is not so much reduced once the elements of the first peak of the s-process are synthesized. Thus, further ncaptures may lead to the second neutron peak $(\mathrm{Ba})$.

We have considered that the s-elements are produced by $(\alpha, n)$ captures on ${ }^{22} \mathrm{Ne}$, as is clearly suggested by the nucleosynthertic models of massive stars at very low metallicities by Frischknecht et al. (2010). We may wonder whether the destruction of ${ }^{13} \mathrm{C}$ by the ${ }^{13} \mathrm{C}(\alpha, n){ }^{16} \mathrm{O}$ reaction in the stages corresponding to Classes 3 may also produce s-elements. If this happens, Classes 3 and 4, which normally contain no s-elements (cf. Table 1), could also present some of them. However, this channel of s-element production should be small, because ${ }^{14} \mathrm{~N}$ is always present at the same time as ${ }^{13} \mathrm{C}$, and it acts like a poison by capturing the free neutrons, thus preventing the production of many s-elements. Nevertheless, some traces of s-elements are possible in all products of He-burning, and this may also be part of why the distributions of $[\mathrm{Sr} / \mathrm{Fe}]$, and $[\mathrm{Ba} / \mathrm{Fe}]$ values are rather broad (Figs. 1, 2).

Finally, we comment on the relations between the $\mathrm{Mg}$ content and the s-elements. The synthesis of ${ }^{25} \mathrm{Mg}$ implies the synthesis of s-elements (at the same time ${ }^{26} \mathrm{Mg}$ is also created). However, at very low $Z$, some small synthesis of ${ }^{24} \mathrm{Mg}$ is also possible, see for example Table 4 by Meynet et al. (2006). Thus, depending on the physical conditions (mass, $Z$, rotation, mass loss, etc.), some small amount of $\mathrm{Mg}$ could be present, without there being any s-elements. Is the opposite possible with some s-elements present without a significant $\mathrm{Mg}$ abundance? This possibility is very marginal, it could occur in the case of a small production of s-elements by $\alpha$-captures on ${ }^{13} \mathrm{C}$.

\section{Proposed classification of CEMP-no stars}

Table 2 summarizes the useful abundance ratios for a classification of the 46 CEMP-no or related stars from Paper I and Hansen et al. (2015). The proposed classes are given in Col. 5. There are clearly not enough data for 16 objects, to which a class cannot be reasonably assigned. A classification appears possible for 30 stars. In several cases and despite some missing data, we have attempted to make the classification. Four cases noted by ":" are very uncertain because of generally insufficient data. We start by the less advanced evolutionary stages.

\subsection{Stars in Classes 2 and $2+$}

This class is characterized by products of He-burning that undergo some first mixing and processing in the H-burning shell and then escape by mass loss. HE0057-5959 shows no sign of excess in $[\mathrm{Ba} / \mathrm{Fe}]$ and $[\mathrm{Sr} / \mathrm{Fe}]$, because this last ratio is very low. $[\mathrm{Mg} / \mathrm{Fe}]$ is close to the mean, but there is a strong excess in the $[\mathrm{Na} / \mathrm{Fe}]$ ratio. $[\mathrm{C} / \mathrm{N}]$ is low, while $[\mathrm{O} / \mathrm{N}]$ is given by an upper limit. ${ }^{12} \mathrm{C} /{ }^{13} \mathrm{C}$ is above 2 . This star shows the effects of $\mathrm{H}-$ burning, and we assign a Class $2+\mathrm{Na}$ to it. This object (like CS 22949-037 in Class 4+) was cataloged as a NEMP-star (nitrogen enhanced metal poor) by Johnson et al. (2007), but it was considered as a CEMP-no star by Norris et al. (2013). We support this last appreciation. Although both Classes 2+ and 4+ have low $[\mathrm{C} / \mathrm{N}]$ values, they also have large differences in parameters, such as $[\mathrm{Sr} / \mathrm{Fe}],[\mathrm{Mg} / \mathrm{Fe}]$, and $[\mathrm{Al} / \mathrm{Fe}]$, which consistently places these two stars in different classes.

HE 1300+0157 is a good example of an object with both very low $[\mathrm{Sr} / \mathrm{Fe}]$ and $[\mathrm{Ba} / \mathrm{Fe}]$ ratios. This is consistent with the low value of the $[\mathrm{Mg} / \mathrm{Fe}]$ ratio. The ratios $[\mathrm{Na} / \mathrm{Fe}]$ and $[\mathrm{Al} / \mathrm{Fe}]$ are well below the mean, and there is certainly no evidence at all of the $\mathrm{Ne}-\mathrm{Na}$ and $\mathrm{Mg}-\mathrm{Al}$ cycles. Both $[\mathrm{C} / \mathrm{N}]$ and $[\mathrm{O} / \mathrm{N}]$ have rather high lower bounds. ${ }^{12} \mathrm{C} /{ }^{13} \mathrm{C}$ is greater than 3 , which is not very constraining. The available data support Class 2 .

HE 1310-0536 shows values close or below the mean for all ratios $[\mathrm{Sr} / \mathrm{Fe}],[\mathrm{Ba} / \mathrm{Fe}],[\mathrm{Na} / \mathrm{Fe}],[\mathrm{Mg} / \mathrm{Fe}]$, and $[\mathrm{Al} / \mathrm{Fe}]$, indicating no excess of s-elements and no evidence of the $\mathrm{Ne}-\mathrm{Na}$ and $\mathrm{Mg}-\mathrm{Al}$ cycles. The ratios $[\mathrm{C} / \mathrm{N}],[\mathrm{O} / \mathrm{N}]$, and ${ }^{12} \mathrm{C} /{ }^{13} \mathrm{C}$ (which we call here "the CNO triplet") are all very low, particularly ${ }^{12} \mathrm{C} /{ }^{13} \mathrm{C}$ and $[\mathrm{C} / \mathrm{N}]$. These various abundances are all consistent with Class $2+$.

HE $1410+0213$ was considered as a low s-star by Allen et al. (2012), however the value of $[\mathrm{Ba} / \mathrm{Fe}]$ is only slightly above the mean (Fig. 2). The CNO triplet has low values, clearly indicating efficient $\mathrm{H}$-burning. That $[\mathrm{Mg} / \mathrm{Fe}]$ is below the mean favors Class 2+, rather than one of the classes of Group 4. However, the data on $\mathrm{Na}, \mathrm{Al}$, and $\mathrm{Sr}$ are missing, and it is difficult to assign a class to this star with confidence. We add the sign of uncertainty.

\subsection{Stars in Classes 3, 3+, and 3++}

In Classes 3,3+, and 3++, we see products that are again mixed into an He-burning region, allowing new elements to experience $\alpha$-captures and to synthesize ${ }^{16} \mathrm{O},\left({ }^{18} \mathrm{O}\right),{ }^{20} \mathrm{Ne},{ }^{22} \mathrm{Ne}$, and possibly ${ }^{24,25,26} \mathrm{Mg}$, as well as s-elements of the first ( $\mathrm{Sr}, \mathrm{Rb}, \mathrm{Y}, \mathrm{Zr}$, etc.) and second ( $\mathrm{Ba}, \mathrm{Cs}, \mathrm{La}, \mathrm{Ce}$, etc.) peaks before they escape in stellar winds.

The star BD +44 493 shows low-to-medium values of all heavy elements $\mathrm{Na}, \mathrm{Mg}, \mathrm{Al}, \mathrm{Sr}$ and $\mathrm{Ba}$ relative to $\mathrm{Fe}$. It has significantly high $[\mathrm{C} / \mathrm{N}]$ and $[\mathrm{O} / \mathrm{N}]$ ratios. The rather high lower limit ${ }^{12} \mathrm{C} /{ }^{13} \mathrm{C}>30$ confirms that Class 3 is appropriate. Radial velocities have been intensively studied, and no significant variations were found for this star (Carney et al. 2003; Norris et al. 2013).

BS 16929-005 has a mean [Ba/Fe], but a high [Sr/Fe], thus it gets a symbol "+". The elements $\mathrm{Na}, \mathrm{Mg}$, and $\mathrm{Al}$ are low. $[\mathrm{C} / \mathrm{N}]$ is moderately high, and ${ }^{12} \mathrm{C} /{ }^{13} \mathrm{C}>7$. These various ratios are clearly consistent with a Class-3+ designation.

CS 22877-001 also does not show excesses of the s-elements, which have medium values. The other heavy elements $\mathrm{Na}, \mathrm{Mg}$, and $\mathrm{Al}$, have low-to-medium values. The rather high $[\mathrm{C} / \mathrm{N}]$ and the lower bound of 10 for ${ }^{12} \mathrm{C} /{ }^{13} \mathrm{C}$ suggests Class 3, however it would be useful to confirm the $\mathrm{O}$ abundance.

$\mathrm{CS} 29502-092$ has a very low $[\mathrm{Ba} / \mathrm{Fe}]$ ratio, while $[\mathrm{Sr} / \mathrm{Fe}]$ is slightly above the mean, but not with significant excess. $\mathrm{Mg}$ and $\mathrm{Al}$ are well below the mean. Data on $\mathrm{Na}$ are missing. The values of $[\mathrm{C} / \mathrm{N}]$ and $[\mathrm{O} / \mathrm{N}]$ are close to 0 , while ${ }^{12} \mathrm{C} /{ }^{13} \mathrm{C}=20$, which is medium-to-high in the context of CEMP-no stars. This star shows the properties of Class 3, but not far from Class 2.

CS 31080-095 has values of $[\mathrm{Sr} / \mathrm{Fe}]$ and $[\mathrm{Ba} / \mathrm{Fe}]$ that are slightly above the mean, however not high enough to give significant excesses (see Figs. 1 and 2). It has very low $\mathrm{Na}$ and $\mathrm{Al}$ content and a value of $[\mathrm{Mg} / \mathrm{Fe}]$ just in the mean. The high $\mathrm{CNO}$ triplet places it in Class 3, not far from $3+$ in view of the $\mathrm{Sr}$ and Ba abundances. That this Class 3 to $3+$ implies the visibility of products of the second He-burning is consistent with the remark by Allen et al. (2012) that it is one of the few O-rich stars with little $\mathrm{Na}$.

HE 0100-1622 shows an excess of [Sr/Fe], but not for barium, so this clearly implies a sign "+". At the same time, it exhibits $[\mathrm{C} / \mathrm{N}]$ much above the mean, also with a positive $[\mathrm{O} / \mathrm{N}]$. Together with the middle-to-high ${ }^{12} \mathrm{C} /{ }^{13} \mathrm{C}=13$, this supports 
Class $3+$. This is consistent with a middle-to-high $\mathrm{Na}$, as well as with evidence of some modest effect of the $\mathrm{Mg}-\mathrm{Al}$ cycle.

For HE 0107-5240, we only have upper bounds for [Ba/Fe] and $[\mathrm{Sr} / \mathrm{Fe}]$ for this very low-metallicity star. Norris et al. (2013) notice that although it cannot be formally classified as a CEMPno star, it shares all its characteristics. In view of the relatively low value of the upper bound for $[\mathrm{Sr} / \mathrm{Fe}]$, this star does not get "+" or "++" symbols. Norris et al. also point out that this star has a long binary period $(P=150 \mathrm{yr})$. Na is slightly above the mean. We notice a high lower bound for ${ }^{12} \mathrm{C} /{ }^{13} \mathrm{C}$, which determines a Class 3. $[(\mathrm{C}+\mathrm{N}+\mathrm{O}) / \mathrm{Fe}]$ is also high. The point in the $[\mathrm{O} / \mathrm{N}]$ vs. $[\mathrm{C} / \mathrm{N}]$ diagram (Paper I), although a bit low, is clearly far from $\mathrm{CNO}$ equilibrium, which is consistent with the presence of products of He-burning.

HE 0134-1519 presents values of $[\mathrm{Sr} / \mathrm{Fe}]$ and $[\mathrm{Ba} / \mathrm{Fe}]$ close to or below the mean. $[\mathrm{Na} / \mathrm{Fe}]$ is much below the mean, and there is also no sign of the $\mathrm{Mg}-\mathrm{Al}$ cycle. The $\mathrm{CNO}$ triplet has only lower bounds. Nevertheless, we propose a Class 3, because this star is neither a Class 4 in view of the low Na nor a Class 2 in view of the average $\mathrm{Sr}$ and $\mathrm{Ba}$ contents. The Class 3 is consistent with a positive $[\mathrm{C} / \mathrm{N}]$, moreover the upper bounds $[\mathrm{N} / \mathrm{Fe}]<1$ and $[\mathrm{O} / \mathrm{Fe}]<2.90$ may also suggest a positive $[\mathrm{O} / \mathrm{N}]$.

HE 0233-0343 clearly has an excess of [ $\mathrm{Sr} / \mathrm{Fe}]$, which implies a "+" sign. For $[\mathrm{Ba} / \mathrm{Fe}]$, we have an upper bound of 0.80 , which allows an excess and thus the possibility of having a "++" object. The data for $\mathrm{Na}, \mathrm{Mg}$ and $\mathrm{Al}$ (upper bound) indicate medium values with no clear evidence of the $\mathrm{Ne}-\mathrm{Na}$ and $\mathrm{Mg}-\mathrm{Al}$ cycles. The lower bound for ${ }^{12} \mathrm{C} /{ }^{13} \mathrm{C}>5$ is not very constraining. The positive $[\mathrm{C} / \mathrm{N}]$ ratio supports Class $3+($ or $3++)$, which is also consistent with the two upper bounds for $[\mathrm{N} / \mathrm{Fe}]<2.80$ and $[\mathrm{O} / \mathrm{Fe}]<4.00$, suggesting a positive $[\mathrm{O} / \mathrm{N}]$ ratio.

HE 0440-1049 is the CEMP-no star with the lowest [C/Fe] ratio (Hansen et al. 2015). It shows a value of $[\mathrm{Sr} / \mathrm{Fe}]$ close to the mean, while clearly $[\mathrm{Ba} / \mathrm{Fe}]$ is very low. $[\mathrm{Na} / \mathrm{Fe}]$ is also low, $[\mathrm{Mg} / \mathrm{Fe}]$ close to the mean, while $[\mathrm{Al} / \mathrm{Fe}]$ is rather low to medium. This indicates an absence of efficient $\mathrm{Ne}-\mathrm{Na}$ and $\mathrm{Mg}-\mathrm{Al}$ cycles. There is a lack of data for the CNO triplet: we only safely know that $[\mathrm{C} / \mathrm{N}]$ is positive, which supports a Class 3 . This agrees with the upper bounds $[\mathrm{N} / \mathrm{Fe}]<0.62$ and $[\mathrm{O} / \mathrm{Fe}]<$ 2.50 , which suggest a positive $[\mathrm{O} / \mathrm{N}]$ ratio.

HE $0557-4840$ has a very low value of $[\mathrm{Sr} / \mathrm{Fe}] .[\mathrm{Ba} / \mathrm{Fe}]$ is given by an upper bound. The values of $[\mathrm{Na} / \mathrm{Fe}],[\mathrm{Mg} / \mathrm{Fe}]$, and $[\mathrm{Al} / \mathrm{Fe}]$ are below the mean, and there is clearly no evidence of the $\mathrm{Ne}-\mathrm{Na}$ and $\mathrm{Mg}-\mathrm{Al}$ cycles. $[\mathrm{C} / \mathrm{N}]$ and $[\mathrm{O} / \mathrm{N}]$, given by lower limits, are largely positive, indicating a dominance of the products of He-burning. ${ }^{12} \mathrm{C} /{ }^{13} \mathrm{C}$ is missing, nevertheless the presently available data support a Class 3 .

HE 1201-1512 shows no evidence of significant s-elements in view of the upper bounds given by Norris et al. (2013), nor of the $\mathrm{Ne}-\mathrm{Na}$ and $\mathrm{Mg}-\mathrm{Al}$ cycles. ${ }^{12} \mathrm{C} /{ }^{13} \mathrm{C}$ is relatively high, and we have a positive lower bound for $[\mathrm{C} / \mathrm{N}]$. These properties allow us to assign a Class 3 to this star.

HE 1506-0113 also presents no excess of s-elements, with values well below the means. $\mathrm{Mg}$ is a bit above the mean, while there is clearly a high $[\mathrm{Na} / \mathrm{Fe}]$ ratio. However, there is no evidence of the $\mathrm{Mg}-\mathrm{Al}$ cycle. ${ }^{12} \mathrm{C} /{ }^{13} \mathrm{C}$ and $[\mathrm{C} / \mathrm{N}]$ are high, while $[\mathrm{O} / \mathrm{N}]$ has a high upper bound. We place this star in Class $3 \mathrm{Na}$.

For HE 2139-5432, both $\mathrm{Sr}$ and $\mathrm{Ba}$ are in the mean or below. $[\mathrm{Na} / \mathrm{Fe}]$ is clearly high, as is $[\mathrm{Mg} / \mathrm{Fe}]$. In addition to the $\mathrm{Ne}-\mathrm{Na}$ cycle, there is an evidence of the operation of the $\mathrm{Mg}-\mathrm{Al}$ cycle, in view of the relatively high value of $[\mathrm{Al} / \mathrm{Fe}] .{ }^{12} \mathrm{C} /{ }^{13} \mathrm{C}$ has a lower limit of 15 , while $[\mathrm{C} / \mathrm{N}]$ and $[\mathrm{O} / \mathrm{N}]$ are above the means. These properties place the star in Class $3 \mathrm{Na}$. That among two stars of Class $3 \mathrm{Na}$, one shows a signature of the $\mathrm{Mg}-\mathrm{Al}$ cycle and the other not may possibly be related to a difference in the mass of the source star, in the sense that the signature of the $\mathrm{Mg}-\mathrm{Al}$ cycle may suggest a higher mass.

Segue $1-7$ shows very low $[\mathrm{Sr} / \mathrm{Fe}]$ and $[\mathrm{Ba} / \mathrm{Fe}]$ ratios. This star has both $[\mathrm{Mg} / \mathrm{Fe}]$ and $[\mathrm{Al} / \mathrm{Fe}]$ somehow above the mean, but not with significant excesses. This might suggest a weak occurrence of the $\mathrm{Mg}-\mathrm{Al}$ cycle. We remark that $[\mathrm{Na} / \mathrm{Fe}]$ is slightly below the mean, however the differences are within about 0.3 dex. It has very high ${ }^{12} \mathrm{C} /{ }^{13} \mathrm{C}$ and $[\mathrm{C} / \mathrm{N}]$ ratios (the upper bound given for $[\mathrm{O} / \mathrm{N}]$ is also high), thus we assign a Class 3 to this star.

The star 53327-2044-515 is considered as closely related to the CEMP-no star by Norris et al. (2013). There is a very strong excess of the ratio $[\mathrm{Sr} / \mathrm{Fe}]$. The upper bound of $[\mathrm{Ba} / \mathrm{Fe}]$ is compatible with a slight excess. Thus, we retain the symbol "+", although it may not be far from "++". There is no evidence of active $\mathrm{Mg}-\mathrm{Al}$ and $\mathrm{Ne}-\mathrm{Na}$ cycles. The lack of data about $[\mathrm{C} / \mathrm{N}]$ and $[\mathrm{O} / \mathrm{N}]$ together with the fact that ${ }^{12} \mathrm{C} /{ }^{13} \mathrm{C}$ only has a lower bound prevents a reliable classification. Provisionally, we suggest a Class $3+$ with a mark of uncertainty.

\subsection{The remarkable stars in Classes 4, 4+, and 4++}

These classes contains stars, where products of He-burning are mixed for the second time (or even more) into the H-burning shell. There is no ambiguity for the definition of Classes 4+ and $4++$. The differences between Classes 4 and $2 \mathrm{Na}$ are small (two classes of mild H-burning), it rests on the very low values of $[\mathrm{Sr} / \mathrm{Fe}]$ and $[\mathrm{Ba} / \mathrm{Fe}]$ in Class 2 and on the higher value of $[\mathrm{Mg} / \mathrm{Fe}]$ in Class 4 . The ratio $[(\mathrm{C}+\mathrm{N}+\mathrm{O}) / \mathrm{Fe}]$ is generally higher in Classes 4, but this is only a statistical indication (see Fig. 7).

CS 22945-017 was considered to be a "b-" star by Allen et al. (2012), which means with $[\mathrm{Ba} / \mathrm{Fe}]$ between 0 and 1 and no europium (r-element). This star shows a relatively high $[\mathrm{Ba} / \mathrm{Fe}]$ ratio, and at the same time relatively low values of the $\mathrm{CNO}$ triplet. Thus, we may consistently put it in Class $4++$, nevertheless indications about $\mathrm{Sr}, \mathrm{Na}$, and $\mathrm{Al}$ would be desirable for a confirmation.

CS 22949-037 shows a low-to-medium value of [Ba/Fe], but a high value of $[\mathrm{Sr} / \mathrm{Fe}]$ with high $[\mathrm{Na} / \mathrm{Fe}]$ and $[\mathrm{Mg} / \mathrm{Fe}]$ ratios. It exhibits low values of the CNO triplet, so that it was sometimes cataloged as a NEMP-star by Johnson et al. (2007), but was considered as a CEMP-no star by Norris et al. (2013). [Al/Fe] is mid to high, which means some operation of the $\mathrm{Mg}-\mathrm{Al}$ cycle. This star corresponds nicely to a class $4+$. We note that despite its s-elements, it presents no enhancement of the heavy neutroncapture elements (of the 2nd and 3rd s-peak) as shown by Norris et al. (2013), which is consistent with our scenario.

The star CS $22958-42$ has a low $[\mathrm{Ba} / \mathrm{Fe}]$, while $[\mathrm{Sr} / \mathrm{Fe}]$ is slighty above the mean. $[\mathrm{Mg} / \mathrm{Fe}]$ and $[\mathrm{Al} / \mathrm{Fe}]$ are below the mean, while $[\mathrm{Na} / \mathrm{Fe}]$ is the highest in the sample; the excess of $\mathrm{Na}$ with respect to $\mathrm{Mg}$ was noted by Allen et al. (2012). In the $[\mathrm{O} / \mathrm{N}]$ vs. $[\mathrm{C} / \mathrm{N}]$ diagram, this object lies on the right side, but a bit low (Maeder et al. 2015), while it is in the main trend of the ${ }^{12} \mathrm{C} /{ }^{13} \mathrm{C}$ vs. $[\mathrm{C} / \mathrm{N}]$ plot. This star well corresponds to a class 4.

CS 29498-043 shares some characteristics of the previous object. $[\mathrm{Sr} / \mathrm{Fe}]$ and $[\mathrm{Ba} / \mathrm{Fe}]$ are close to the mean, with more $\mathrm{Mg}$ and $\mathrm{Al}$, thus providing some indications of the activity of the $\mathrm{Mg}-\mathrm{Al}$ cycle. $[\mathrm{Na} / \mathrm{Fe}]$ is marginally in excess. The $\mathrm{CNO}$ triplet is low to mid. We also assign a class 4 . The absence of heavy neutron-capture elements was also emphasized, as for CS 22949-037 (Norris et al. 2013).

We now turn to CS 30322-023 a star which shows a CNO triplet remarkably corresponding to nearly pure CNO equilibrium (Paper I). At the same time, it presents a relatively 
high Ba content and was cataloged as "b-" by Allen et al. (2012), this places it clearly in class $4++$. Both $[\mathrm{Mg} / \mathrm{Fe}]$ and $[\mathrm{Na} / \mathrm{Fe}]$ are above the mean. The problem is that the gravity of this star is very low. According to Masseron et al. (2006) this star may be a TP-AGB star, a point of view which we support. Thus, in this case many of its properties could result from self-enrichment, nevertheless according to these authors it shows no evidence of europium. We keep it as a CEMP-no star and assign a class 4++ to it.

HE 1012-1540 has mean values of $[\mathrm{Sr} / \mathrm{Fe}]$ and $[\mathrm{Ba} / \mathrm{Fe}]$. It clearly shows strong evidences of the $\mathrm{Ne}-\mathrm{Na}$ and $\mathrm{Mg}-\mathrm{Al}$ cycles. The $[\mathrm{C} / \mathrm{N}]$ and $[\mathrm{O} / \mathrm{N}]$ ratios are rather high, clearly showing the signature of He-burning. Thus we assign a class $3 \mathrm{Na}$ to this object. In view of its $\mathrm{Mg}$ and $\mathrm{Al}$ abundances, it may not be far from a class 4 . The ${ }^{12} \mathrm{C} /{ }^{13} \mathrm{C}$ data, which would be most useful, are not available.

HE 1327-2326 was for some time the most iron-poor star known (Frebel et al. 2005), it has only a (high) upper limit $[\mathrm{Ba} / \mathrm{Fe}]<1.46$, thus it cannot formally by classified as a CEMPno star, although it shares the other characteristics of this class (Norris et al. 2013). However, we have seen that stars showing some $\mathrm{Sr}$ and $\mathrm{Ba}$ may belong to the genetic family of CEMPno stars, i.e. their abundances result from the same processes of mixing and mass loss as the other CEMP-no stars. The [ $\mathrm{Sr} / \mathrm{Fe}]$ ratio is the highest in the sample. The contents in $\mathrm{Na}, \mathrm{Mg}$ and $\mathrm{Al}$ are also very high. At the same time, the CNO triplet is clearly low, thus providing a dominant signature of $\mathrm{H}$-burning, while $[(\mathrm{C}+\mathrm{N}+\mathrm{O}) / \mathrm{Fe}]$ is quite large. Without any ambiguity, we assign a class $4++$ to it. We would say that HE 1327-2326 is an outstanding illustration of our scenario for explaining the abundances of CEMP-no stars.

HE 1419-1324 shows a strong excess of [Ba/Fe], which in itself support a " ++ " symbol. It is recognized as a low-s star (Masseron et al. 2010; Allen et al. 2012). There is no data about $\mathrm{Sr}$ and $\mathrm{Na} .[\mathrm{Mg} / \mathrm{Fe}]$ is close to the mean. $[\mathrm{C} / \mathrm{N}]$ and $[\mathrm{O} / \mathrm{N}]$ are below the mean, while the ratio ${ }^{12} \mathrm{C} /{ }^{13} \mathrm{C}$ is medium. It is likely a Class-4++ star, but the lack of data about $\mathrm{Sr}, \mathrm{Na}$, and $\mathrm{Al}$ makes the identification incomplete, hence the symbol ":".

HE 2331-7155 shows low-to-moderate values of [Sr/Fe] and $[\mathrm{Ba} / \mathrm{Fe}]$. The ratio $[\mathrm{Na} / \mathrm{Fe}]$ is slightly lower than the median value. The ratio $[\mathrm{Mg} / \mathrm{Fe}]$ is high, while $[\mathrm{Al} / \mathrm{Fe}]$ is in the mean. The CNO triplet is low, especially $[\mathrm{C} / \mathrm{N}]=-1.23$. This is a clear signature of $\mathrm{H}$-burning, which favors Class $2+$ or 4 . On the other hand, the moderate values of $\mathrm{Sr}$ and $\mathrm{Ba}$ and the high values of $\mathrm{Mg}$ indicate the possible previous contribution of He-burning. Thus, we tend to assign Class 4 to this star.

The presently most Fe-poor star known, SMSS 0313-6708, cannot be classified safely at present, since it essentially has upper bounds for its very weak abundances of heavy elements. This is not the case for $\mathrm{Mg}$, and we have $[\mathrm{Mg} / \mathrm{Fe}]>3.3$. This extremely high value tends to imply a very heavy He-burning (labeled with ++ ). This is consistent with the relatively high upper bounds for $[\mathrm{Sr} / \mathrm{H}]<-6.7$ and $[\mathrm{Ba} / \mathrm{H}]<-6.1 \mathrm{com}-$ pared to $[\mathrm{Fe} / \mathrm{H}]<-7.1$, as obtained by Keller et al. (2014). Interestingly enough, in Paper I we could have both an upper bound $(-2.49)$ and a lower bound $(-3.09)$ for $[(\mathrm{C}+\mathrm{N}+\mathrm{O}) / \mathrm{H}]$. Adopting the mean $[(\mathrm{C}+\mathrm{N}+\mathrm{O}) / \mathrm{H}] \approx-2.8$, we obtain a lower bound $[(\mathrm{C}+\mathrm{N}+\mathrm{O}) / \mathrm{Fe}]>4.3$ (which is used in Figs. 6 and 7). The lower limit ${ }^{12} \mathrm{C} /{ }^{13} \mathrm{C}>4.5$ may allow some H-burning. Therefore, this extreme object could belong to Class $4++$, but it is uncertain in view of the difficulty of obtaining better data for this extreme object.

We note that the uncertain cases essentially result from our having tried, rightly or wrongly, to still classify a few stars with

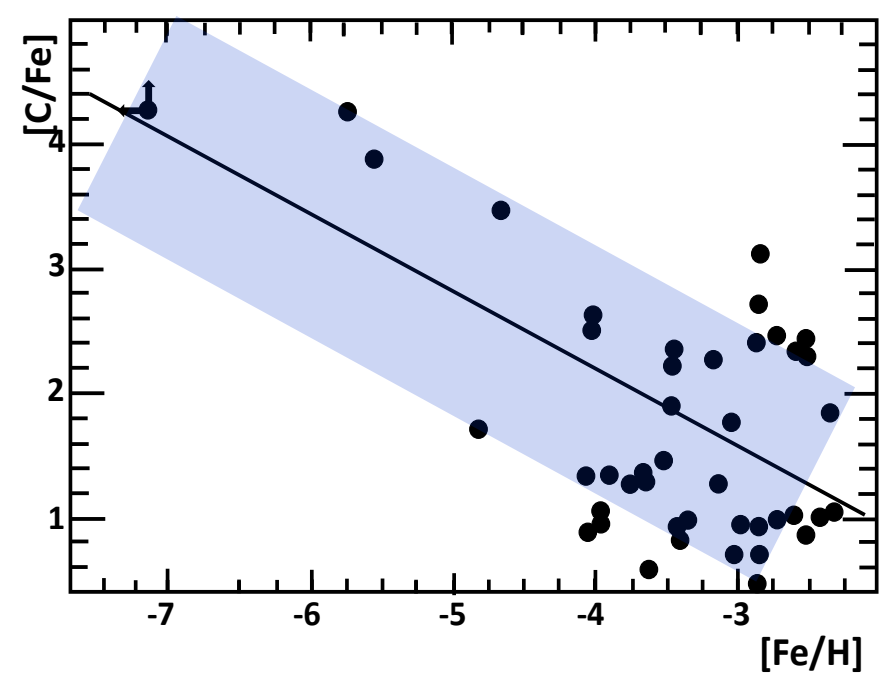

Fig. 6. Rather poor relation between $[\mathrm{C} / \mathrm{Fe}]$ and $[\mathrm{Fe} / \mathrm{H}]$ for $\mathrm{CEMP}-\mathrm{no}$ stars. The light blue band indicates the standard deviation in $[\mathrm{C} / \mathrm{Fe}]$ of 0.99 on both sides of the least square average relation.

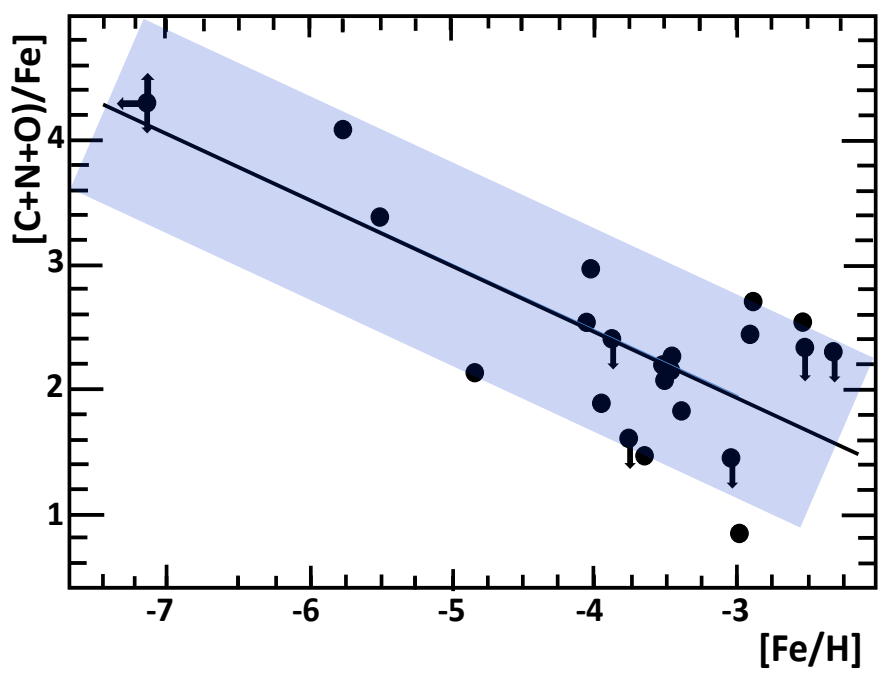

Fig. 7. Relation between $[(\mathrm{C}+\mathrm{N}+\mathrm{O}) / \mathrm{Fe}]$ and $[\mathrm{Fe} / \mathrm{H}]$ for CEMP-no stars. The light blue band indicates the standard deviation in $[(\mathrm{C}+\mathrm{N}+\mathrm{O}) / \mathrm{Fe}]$ of 0.88 on both sides of the least square average relation.

marginally insufficient data. There are only a few stars in the group of Classes 2, compared to the groups of Classes 3 and 4. This may indicate that there are in general a few back-and-forth partial mixing motions between the $\mathrm{H}$ - and He-burning zones. The main result is that the stars with sufficient data can generally be classified well in the proposed system. The various classes cover the observed variety of the chemical abundances of CEMP-no stars. This supports the view that the effects of mixing (likely due to rotation) and mass loss are responsible for the variety of composition in CEMP-no stars.

\subsection{Behavior as a function of $[\mathrm{Fe} / \mathrm{H}]$}

CEMP-no stars span a range of more than $4.5 \mathrm{dex}$ in $[\mathrm{Fe} / \mathrm{H}]$ and $[\alpha$-elements/Fe]. (We have seen in Paper I that the $\alpha$-elements, with an atomic mass number above 24 such as $\mathrm{Ca}$ and $\mathrm{Si}$, behave like $[\mathrm{Fe} / \mathrm{H}]$.) We now examine whether some trends appear over this broad range of metallicities. 
In each class, there are stars with a wide variety of $[\mathrm{Fe} / \mathrm{H}]$ values. This is not surprising since at each metallicity, there is probably a variety of rotation velocities and probably also of mass loss rates. Nevertheless, it seems that there is a trend toward the higher classes appearing at lower $[\mathrm{Fe} / \mathrm{H}]$. For Classes 2, the average $[\mathrm{Fe} / \mathrm{H}]=-3.63$; for Classes 3 , it is -3.72 ; and for Classes 4 , the average is -3.98 . Certainly, more data are needed to ascertain this trend based on a small sample, which may be influenced by a few extreme values.

The main criteria for CEMP stars are the $[\mathrm{C} / \mathrm{Fe}]$ ratios, which we now examine. A plot of $[\mathrm{C} / \mathrm{Fe}]$ vs. $[\mathrm{Fe} / \mathrm{H}]$ for the stars in Table 2 is shown in Fig. 6, which also indicates the standard deviation. Such an essential plot was also shown by Norris et al. (2013). We see that there is a trend, but the relation is poor. Without the four stars on the left, there would be no trend at all. The scatter around the average relation is large, amounting to about 1 dex. We have a slightly better relation when we correlate $[(\mathrm{C}+\mathrm{N}+\mathrm{O}) / \mathrm{Fe}]$ with $[\mathrm{Fe} / \mathrm{H}]$ in Fig. 7 .

We may understand why the relation of $[(\mathrm{C}+\mathrm{N}+\mathrm{O}) / \mathrm{Fe}]$ vs. $[\mathrm{Fe} / \mathrm{H}]$ is better than for $[\mathrm{C} / \mathrm{Fe}]$. This may be because the sum of $\mathrm{C}+\mathrm{N}+\mathrm{O}$ elements only results from the number of products (mainly $\mathrm{C}$ and $\mathrm{O}$ ) of $\mathrm{He}$-burning that migrates into the $\mathrm{H}$-burning region; this amount is not modified by the $\mathrm{CNO}$ reactions in the source star. As to $\mathrm{C}$, its abundance is influenced by the strength of both the mixing process and the CNO-burning in the source stars.

In this respect, we note that the individual $\mathrm{C}, \mathrm{N}$, and $\mathrm{O}$ abundances may also be modified by the $\mathrm{CNO}$ processing in the CEMP-no stars themselves. The evolutionary effects of CNO processing on the carbon abundances have been been studied in detail as a function of $\log g$ by Placco et al. (2014), with the interesting result that it allows them to apply a correction on the $\mathrm{C}$ abundances of CEMP stars. This leads to a much better estimate of the fraction of these stars as a function of $[\mathrm{Fe} / \mathrm{H}]$. We may point out that the effects studied by Placco et al. (2014) bring essentially no change for the sum $[(\mathrm{C}+\mathrm{N}) / \mathrm{Fe}]($ or $[(\mathrm{C}+\mathrm{N}+\mathrm{O}) / \mathrm{Fe}])$, while they evidently change $[\mathrm{C} / \mathrm{Fe}]$.

The relation between $[(\mathrm{C}+\mathrm{N}+\mathrm{O}) / \mathrm{Fe}]$ and $[\mathrm{Fe} / \mathrm{H}]$ may suggest that this is essentially the higher strength of the mixing (and maybe the higher rotation) in the source stars at lower $[\mathrm{Fe} / \mathrm{H}]$, which is responsible for the peculiarities of the CEMPno stars, where the exchanges between the two burning regions are more intense at lower $[\mathrm{Fe} / \mathrm{H}]$. This higher effect of mixing at lower $\mathrm{Z}$ was first found (and explained) in the context of stellar models at SMC composition (Maeder \& Meynet 2001). It was then confirmed by studying the origin of primary nitrogen in low-metallicity galaxies (Meynet \& Maeder 2002), after the evidence of primary nitrogen had firs beent been demonstrated by Edmunds \& Pagel (1978). That we observe strong mixing effects in CEMP-no stars indicates that mixing continues to grow at much lower metallicities than for the SMC.

\section{Conclusions}

The classes we proposed on the basis of the nucleosynthesis in successive phases of mixing between the $\mathrm{H}$ - and He-burning regions cover the great variety of $\mathrm{C}, \mathrm{N}, \mathrm{O}, \mathrm{Na}, \mathrm{Mg}$, and $\mathrm{Al}$ abundances observed in CEMP-no stars well. The classification is essentially model independent. A few transition and uncertain cases appear mainly by insufficient data, but there is no dichotomy between the classes considered and the variety of abundances observed. A possible extension of this classification to the other types of CEMP stars with $[\mathrm{Fe} / \mathrm{H}]$ higher than $\approx-2.5$ could be envisaged in future.
We may wonder at what stage in the evolution of the massive stars does occur the matter ejection leading to CEMP-no stars. There are four stars in Classes 2, 17 in Classes 3 and 9 in Classes 4. The total of 13 stars ejected in Classes 2 and 4 show the signatures of $\mathrm{CNO}$ processing, with the last nuclear reactions having occurred before the ejection (with more turnovers in Classes 4 than in 2). This means that the H-burning shell was still active in the outer stellar layers just before the ejection. In terms of evolution, this points clearly toward ejection during the supergiant stages, whether blue or red.

The situation is different for the 17 stars in Classes 3. There, the main nuclear signature before the ejection is that of Heburning. This necessarily implies that the H-burning shell was nearly or totally extinct at the time of large mass ejections. This usually occurs very late in the evolution, shortly before the supernova explosion. This may correspond to the suggestion by Moriya \& Langer (2015) that very intense mass ejections due to pulsational instabilities occur in the red supergiant stage of metal-free stars shortly before the supernova explosions, thus creating very dense circumstellar shells. Another possibility is that of mass ejection at advanced stages for homogeneous evolution due to mixing, a situation which may occur in very fastrotating massive stars (Maeder 1987).

On the whole, this classification, along with the tests we performed in Paper I support the view that rotating massive stars with mixing and mass loss strongly influenced the early chemical (and probably spectral) evolution of galaxies.

Acknowledgements. We express our deepest thanks to our friends and colleagues Corinne Charbonnel, Patrick Eggenberger, Sylvia Ekstroem, and Arthur Choplin for very useful discussions. We also thank Prof. Timothy Beers for providing new data in advance of publication and for very helpful remarks.

\section{Appendix A: CEMP-no stars that cannot be classified}

There are 16 stars that we cannot classify because some essential chemical abundances are not available. They deserve some brief comments. For CS 22166-016, the values of [Ba/Fe] and $[\mathrm{Sr} / \mathrm{Fe}]$ show no excesses, the same for $[\mathrm{Na} / \mathrm{Fe}]$ and $[\mathrm{Mg} / \mathrm{Fe}]$. These values imply that it is not in Class $4,4+, 4++, 3+$, or $3++$. However, the lack of the CNO triplet prevents us from deciding whether it belongs to Class 2 or 3. For CS 22878-027, there is no evidence of significant excess of s-elements, and the absence of data for the CNO triplet also prevents the classification (only a non-constraining lower limit for $[\mathrm{C} / \mathrm{N}]$ is available). CS 22885 096 has a low $[\mathrm{C} / \mathrm{Fe}]=0.60$ (Norris et al. 2001), which is a marginally low value for a CEMP-no star. It is, however, considered to be a CEMP-no star by Allen et al. (2012). The lack of the CNO triplet and of $[\mathrm{Na} / \mathrm{Fe}]$ does not allow us to assign a class to this star, which has extremely low values of $[\mathrm{Sr} / \mathrm{Fe}]$ and $[\mathrm{Ba} / \mathrm{Fe}]$. CS 22956-028 is a binary star discussed by Sneden et al. (2003), and it is considered to be a low-s star by Masseron et al. (2010). The $[\mathrm{Ba} / \mathrm{Fe}]$ ratio of 0.16 is above the mean, but it is not a clear excess according to Fig. 2. The lack of the Sr- and Na-abundances prevents any reliable classification. CS 22957027 shows radial velocity variations (Preston \& Sneden 2001; Norris et al. 2013), it is probably in Class 2 in view of the ratio ${ }^{12} \mathrm{C} /{ }^{13} \mathrm{C}$, but it cannot be confirmed owing to the lack of $\mathrm{O}-$ and Na-data. CS 30314-067 has values of [ $\mathrm{Sr} / \mathrm{Fe}]$ close to the mean and of $[\mathrm{Ba} / \mathrm{Fe}]$ slightly below the mean. $[\mathrm{Mg} / \mathrm{Fe}]$ and $[\mathrm{Al} / \mathrm{Fe}]$ are in the mid range, while $[\mathrm{Na} / \mathrm{Fe}]$ is much lower than the mean. There is no clear evidence of the $\mathrm{Ne}-\mathrm{Na}$ and $\mathrm{Mg}-\mathrm{Al}$ cycles. The low value of $[\mathrm{C} / \mathrm{N}]$ is a signature of efficient H-burning. We 
would place it in Class 4, however the lack of $[\mathrm{O} / \mathrm{N}]$ and ${ }^{12} \mathrm{C} /{ }^{13} \mathrm{C}$ prevents any confirmation and leaves open the possibility of a Class $2+$. For G 77-61, the high upper limit for $[\mathrm{Ba} / \mathrm{Fe}]$ is not constraining and does not allow us to determine a class for this star.

For HE $0007-1832$, the ratio $[\mathrm{Ba} / \mathrm{Fe}]$ is at the limit for showing an excess, $[\mathrm{C} / \mathrm{N}]$ is well above the mean, however the available data are clearly insufficient to allow a classification. $\mathrm{HE}$ 0146-1548 has a value of $[\mathrm{Ba} / \mathrm{Fe}]$ below the mean and $[\mathrm{Sr} / \mathrm{Fe}]$ is close to the mean. $[\mathrm{Mg} / \mathrm{Fe}]$ is above the mean, and there is some marginal indications of the $\mathrm{Ne}-\mathrm{Na}$ and $\mathrm{Mg}-\mathrm{Al}$ cycles. These properties, together with the low ${ }^{12} \mathrm{C} /{ }^{13} \mathrm{C}$ ratio, correspond to a Class 4 or $4+$, however the absence of values for $[\mathrm{C} / \mathrm{N}]$ and $[\mathrm{O} / \mathrm{N}]$ prevents a safe assignation. For HE 1150-0428, $[\mathrm{Ba} / \mathrm{Fe}]$ has a mean value, while $[\mathrm{Sr} / \mathrm{Fe}]$ is slightly above the mean, thus there is no evidence of significant enhancements in s-elements. $[\mathrm{Mg} / \mathrm{Fe}]$ is below the mean. There is no information about the $\mathrm{Ne}-\mathrm{Na}$ and $\mathrm{Mg}-\mathrm{Al}$ cycles. From the low ${ }^{12} \mathrm{C} /{ }^{13} \mathrm{C}$ and $[\mathrm{C} / \mathrm{N}]$ ratios, we would propose a Class $2+$ or 4 , but we have not enough data to decide. For HE 1300-0641, a lot of data is missing and even a tentative classification is impossible. We can only say that there is no indication of excesses for s-elements. $[\mathrm{Mg} / \mathrm{Fe}]$ is low, and in view of $[\mathrm{Al} / \mathrm{Fe}]$, the $\mathrm{Mg}-\mathrm{Al}$ cycle has certainly not operated. HE 1300-2201 presents a significant enrichment in $[\mathrm{Sr} / \mathrm{Fe}]$, but $[\mathrm{Ba} / \mathrm{Fe}]$ is only slightly above the mean. The value of $[\mathrm{Mg} / \mathrm{Fe}]$ is below the mean, and there is no evidence of the $\mathrm{Mg}-\mathrm{Al}$ cycle. Although this star has no more data than the previous one, the significant $[\mathrm{Sr} / \mathrm{Fe}]$ excess is somehow constraining. The class is probably $3+$, nevertheless the lack of the CNO triplet and of $[\mathrm{Na} / \mathrm{Fe}]$ prevents a reliable classification. HE 1330-0354 shows no Ba excess, but $[\mathrm{Sr} / \mathrm{Fe}]$ is just above the limit of what we consider as an excess. It has little $\mathrm{Mg}$ and $\mathrm{Al}$. Thus, it could be in class 3+, however the lack of data makes this possibility unconfirmed. HE 2142-5656 does not show enhancements of s-elements, neither of the occurrence of $\mathrm{Ne}-\mathrm{Na}$ and $\mathrm{Mg}-\mathrm{Al}$ cycles. All values are not far from the means. The same is true for $[\mathrm{C} / \mathrm{N}]$, however the lack of $[\mathrm{O} / \mathrm{N}]$ and ${ }^{12} \mathrm{C} /{ }^{13} \mathrm{C}$ ratios prevents an unambiguous classification, $\mathrm{HE}$ 2202-4831 also shows no evidence of excesses of s-elements, the values of $[\mathrm{Ba} / \mathrm{Fe}]$ and $[\mathrm{Sr} / \mathrm{Fe}]$ being clearly below the means. $\mathrm{Mg}$ is below the mean, while $[\mathrm{Na} / \mathrm{Fe}]$ is above the mean, close to the limit of having an excess. The absence of data on the CNO triplet prevents us to assign a class. For HE 2247-7400, the situation is about the same: no sign of excess of s-elements, both $\mathrm{Mg}$ and $\mathrm{Na}$ below the mean. Here also, the absence of the CNO triplet does not allow a classification.

\section{References}

Allen, D. M., Ryan, S. G., Rossi, S., Beers, T. C., \& Tsangarides, S. A. 2012, A\&A, 548, A34

Beers, T. C., \& Christlieb, N. 2005, ARA\&A, 43, 531

Beers, T. C., Preston, G. W., \& Shectman, S. A. 1992, AJ, 103, 1987

Carney, B. W., Latham, D. W., Stefanik, R. P., Laird, J. B., \& Morse, J. A. 2003, AJ, 125, 293

Chiappini, C., Ekström, S., Meynet, G., et al. 2008, A\&A, 479, L9

Crowther, P. A. 2010, Hot Massive Stars: The Impact of HST, ed. F. D. Macchetto, 3

Cyburt, R. H., Fields, B. D., \& Olive, K. A. 2008, J. Cosmol. Astropart. Phys., 11,12

Edmunds, M. G., \& Pagel, B. E. J. 1978, MNRAS, 185, 77P

Frebel, A., Aoki, W., Christlieb, N., et al. 2005, Nature, 434, 871

Frischknecht, U., Hirschi, R., Meynet, G., et al. 2010, A\&A, 522, A39

Hansen, T., Hansen, C. J., Christlieb, N., et al. 2015, ApJ, 807, 173

Ito, H., Aoki, W., Beers, T. C., et al. 2013, ApJ, 773, 33

Johnson, J. A., Herwig, F., Beers, T. C., \& Christlieb, N. 2007, ApJ, 658, 1203

Keller, S. C., Bessell, M. S., Frebel, A., et al. 2014, Nature, 506, 463

Lebouteiller, V., Heap, S., Hubeny, I., \& Kunth, D. 2013, A\&A, 553, A16

Maeder, A. 1987, A\&A, 178, 159

Maeder, A. 2009, Physics, Formation and Evolution of Rotating Stars (Berlin, Heidelberg: Springer)

Maeder, A., \& Meynet, G. 2001, A\&A, 373, 555

Maeder, A., \& Meynet, G. 2012, Rev. Mod. Phys., 84, 25

Maeder, A., Meynet, G., \& Chiappini, C. 2015, A\&A, 576, A56 (Paper I)

Masseron, T., van Eck, S., Famaey, B., et al. 2006, A\&A, 455, 1059

Masseron, T., Johnson, J. A., Plez, B., et al. 2010, A\&A, 509, A93

Matteucci, F. 2012, Chemical Evolution of Galaxies (Berlin, Heidelberg: Springer)

Meynet, G., \& Maeder, A. 2002, A\&A, 381, L25

Meynet, G., Ekström, S., \& Maeder, A. 2006, A\&A, 447, 623

Moriya, T. J., \& Langer, N. 2015, A\&A, 573, A18

Nomoto, K., Kobayashi, C., \& Tominaga, N. 2013, ARA\&A, 51, 457

Norris, J. E., Ryan, S. G., \& Beers, T. C. 2001, ApJ, 561, 1034

Norris, J. E., Yong, D., Bessell, M. S., et al. 2013, ApJ, 762, 28

Placco, V. M., Frebel, A., Beers, T. C., \& Stancliffe, R. J. 2014, ApJ, 797, 21

Preston, G. W., \& Sneden, C. 2001, AJ, 122, 1545

Sneden, C., Preston, G. W., \& Cowan, J. J. 2003, ApJ, 592, 504 